\title{
Artroscopia em cães
}

\section{Arthroscopy in dogs}

\section{Artroscopia en perros}

\section{Angélica Cecília Tatarunas'구 Julia Maria Matera²}

Departamento de Cirurgia da Faculdade de Medicina Veterinária e Zootecnia da Universidade de São Paulo. São Paulo, SP, Brasil

\section{Resumo}

Objetivo: Apresentar as vantagens e desvantagens do uso da artroscopia, treinamento, instrumental, técnica e complicações, bem como particularidades e indicações do procedimento nas articulações do ombro, joelho, cotovelo, tarso, coxofemoral e carpo. Fontes Pesquisadas: CAB e MEDLINE, período retrospectivo de 24 anos. Síntese dos Dados: Apesar de a artroscopia ser amplamente utilizada para o diagnóstico e tratamento das afecções articulares no homem e no cavalo, somente nos últimos anos é que ela vem se tornando relevante na espécie canina. Ela permite um exame direto realizado sob magnificação da imagem e iluminação da cartilagem, membrana sinovial e demais estruturas intra-articulares. Conclusões: Devido ao seu caráter pouco invasivo, a artroscopia promove menor trauma tecidual, retorno mais rápido da função do membro e melhor resultado cosmético.

Palavras-chave: Artroscopia. Articulações, cirurgia. Artroscópios. Cães. 
Tatarunas, A. C.; Matera, J. M. Artroscopia em cães. / Arthroscopy in dogs. / Artroscopia en perros. Rev. Educ. Contin. CRMV-SP /Contin. Educ. J. CRMV-SP, São Paulo, v. 6, n. 1/3, p. 63-73, 2003.

\section{Introdução}

A artroscopia, que consiste na inspeção das estruturas intra-articulares por meio de um instrumento óptico (van BREE; van RYSSEN ${ }^{1}$, 1998), é classicamente utilizada na ortopedia para o diagnóstico e tratamento das afecções articulares no homem $\left(\right.$ ROCKWOOD $\left.^{2}, 1988\right)$ e no cavalo (JOHNSON; HULSE ${ }^{3}, 2002$; McILWRAITH; McCRACKEN $\left.{ }^{4}, 1990\right)$.

A despeito da evolução da artroscopia no homem e no cavalo, na espécie canina o seu desenvolvimento foi lento e somente nos últimos anos é que ela vem se tornando relevante. A cirurgia artroscópica nos pequenos animais e o seu estudo em articulações menores é ainda mais recente (BEALE et al. ${ }^{5}$, 2003; ROCHAT $^{6}, 2001$; TAYLOR 7 , 1999). O espaço limitado das pequenas articulações caninas, o custo do equipamento e o fato de uma artrotomia não ser tão dramática para o cão como é para o homem, tanto em morbidade como em resultados cosméticos, são citados como causas prováveis ao retardo do seu desenvolvimento nesta espécie ${ }^{1,5}$.

No cão, semelhante ao homem, os relatos iniciais referiam-se à articulação do joelho (KIVUMBI; BENNETT $^{8}$, 1981; SIEMERING $^{9}$, 1978). Em 1986 Person ${ }^{10}$ publicou estudo sobre a abordagem artroscópica do ombro e, em $1989^{11}$, da articulação coxofemoral, concluindo que esta última forneceu avaliação completa, eficiente e atraumática. Os primeiros relatos da articulação do cotovelo e articulação do tarso couberam a van Ryssen et al. ${ }^{12,13}$ no ano de 1993.

Os procedimentos cirúrgicos primeiramente realizados no cão via artroscopia foram a reconstrução do ligamento cruzado cranial (PERSON $\left.{ }^{14}, 1987\right)$ e o tratamento de osteocondrite dissecante da articulação do ombro (PERSON ${ }^{15}$, 1989). Nessa espécie, a artroscopia diagnóstica e cirúrgica vem sendo estudada principalmente nas articulações do ombro, joelho e cotovelo; e, com menor ênfase, nas articulações do tarso, coxofemoral e carpo ${ }^{5}$.

A presente revisão teve o intuito de apresentar as vantagens e desvantagens do uso da artroscopia, treinamento, instrumental, técnica e complicações, bem como particularidades e indicações do procedimento nas articulações do ombro, joelho, cotovelo, tarso, coxofemoral e carpo. Os dados bibliográficos foram obtidos por meio do CAB e MEDLINE, abrangendo um período retrospectivo de 24 anos.

\section{Vantagens e Desvantagens}

A artroscopia fornece um exame direto da cartilagem articular, membrana sinovial, meniscos e ligamentos intra-articulares ${ }^{4,10,16}$. Devido à magnificação da imagem e concomitante iluminação, a artroscopia permite uma qualidade na visibilidade das estruturas intra-articulares superior à da artrotomia (MILLER; PRESNELL $^{17}$, 1985; van GESTEL $\left.{ }^{18}, 1985\right)$.

O caráter minimamente invasivo do procedimento artroscópico viabiliza a intervenção em mais de uma articulação em um mesmo procedimento cirúrgico (van RYSSEN; van BREE $\left.{ }^{19}, 1997\right)$. As pequenas incisões dos portais, quando comparadas com à de uma artrotomia, pela cápsula articular inflamada, seccionam menor número de terminações nervosas, minimizando o estímulo da dor no pós-operatório ${ }^{5}$.

Durante a artroscopia, as vilosidades sinoviais são observadas em meio líquido, portanto a sua morfologia é característica, em contrapartida à artrotomia, em que elas estão colabadas e não podem ser tão bem analisadas. Alterações pertinentes à vascularização (hiperemia e petéquias), proliferação, morfologia e localização dos vilos podem ocorrer nas sinovites ${ }^{8,20}$, evidenciando as várias afecções articulares ${ }^{4}$.

Vantagens da artroscopia cirúrgica sobre a artrotomia convencional incluem melhor visibilidade, menor morbidade e precisão otimizada ${ }^{5}$, bem como menor trauma tecidual, retorno mais rápido da função e melhor resultado cosmético ${ }^{8}$.

Desvantagens da artroscopia incluem o custo relativamente alto do equipamento, a longa curva de aprendizado, continuidade da prática e necessidade de uma equipe cirúrgica treinada ${ }^{5,7,19,21,22}$. Em alguns casos, a artroscopia pode ser insatisfatória e terminar em uma artrotomia, o que prolonga a intervenção ${ }^{17}$.

A artroscopia não é uma técnica fácil; ela requer paciência, treinamento e persistência para for- 
necer bons resultados ${ }^{9,23}$. O pequeno confinamento ósseo rígido, a complexidade de algumas articulações e a fragilidade dos instrumentos tornam a artroscopia a técnica endoscópica rígida de maior dificuldade ${ }^{6,16}$.

Uma fase pertinente ao aprendizado é o prévio treinamento em cadáveres, com confirmação das alterações observadas por artroscopia e das lesões produzidas iatrogenicamente pelo exame necroscópico (PER$\left.\mathrm{SON}^{24}, 1985\right)$. Neste estágio deve-se obter um alto nível de proficiência técnica e experiência no reconhecimento das estruturas intra-articulares normais e alteradas $^{8}$.

\section{Instrumental Artroscópico}

O artroscópio é um telescópio de diâmetro fino, com uma porção externa de fibras ópticas que transporta luz para dentro da articulação e uma série de lentes internas que conduzem a imagem ao olho ou câmera ${ }^{5}$. Ele está disponível em diferentes diâmetros, comprimentos e ângulos de lente ${ }^{3}$.

O diâmetro do artroscópio corresponde à circunferência externa da porção tubular sem a cânula. Nos cães, utilizam-se diâmetros de 1,9 mm a 4,0 mm; sendo o de 2,7 mm o mais usado nesta espécie ${ }^{5,16,21}$.

O comprimento de uso no trabalho refere-se ao comprimento total do artroscópio sendo denominado curto, com cerca de $8,5 \mathrm{~cm}$, ou longo, em torno de 13 $\mathrm{cm}$. Ambos são utilizados no cão: o curto é indicado para articulações com menor massa muscular, como o cotovelo $^{6,21}$ e o longo para articulações mais profundas como a do ombro 5 .

O artroscópio pode ter a sua extremidade reta ou angulada. A presença da angulação permite que o campo visual seja aumentado unicamente pela sua rotação. No cão utiliza-se geralmente uma lente com ângulo de $30^{\circ} 1,3,6,25$.

$\mathrm{O}$ artroscópio é inserido e manipulado dentro da articulação através da cânula, a qual tem a função de proteção ${ }^{3}$. $\mathrm{O}$ espaço existente entre a cânula e o telescópio permite que haja fluxo de fluido para a articulação, controlado por um sistema de torneiras ${ }^{1,3}$.
A cânula é posicionada no interior da articulação com a ajuda do trocarte, o qual pode ser agudo ou rombo. O trocarte agudo é raramente utilizado e se presta principalmente para a perfuração da porção fibrosa da cápsula articular, enquanto que o rombo pode ser usado para perfurar a cápsula articular em toda a sua espessura e modificar a posição da cânula no interior da articulação ${ }^{1}$.

A visualização, da articulação é realizada por observação direta ou por vídeoartroscopia, em que há a transferência da imagem para um monitor de vídeo. A vídeoartroscopia fornece magnificação superior da imagem, possibilita que várias pessoas acompanhem o procedimento, permite gravação e revisão, diminui a chance de contaminação e facilita a performance do procedimento cirúrgico (PERSON ${ }^{15}, 1989$; TOMLINSON $^{21}$, 2001).

Para a iluminação, geralmente emprega-se a luz de xenônio, branca e brilhante ${ }^{6}$, com intensidade entre $100 \mathrm{~W}$ e $400 \mathrm{~W}^{\urcorner}$. O artroscópio é conectado a uma fonte de luz por um cabo de fibra óptica, o qual transfere a luminosidade para dentro da articulação sem aquecimento ${ }^{21}$.

A articulação deve ser mantida distendida durante todo o procedimento artroscópico, o que pode ser feito com gás ou com líquido (ERIKSON; SEBIK ${ }^{26}$, 1982), sendo utilizada solução salina ou ringer lactato ${ }^{1}$.

É necessário fluxo de líquido adequado para expandir a articulação e permitir uma boa visualização do campo operatório, o qual é obtido com o estabelecimento de portais de irrigação e drenagem. O líquido pode ser injetado por gravidade associado ou não, por uma bolsa de pressão ou ainda por um sistema de bomba de infusão ${ }^{3,23}$.

Instrumentos utilizados durante artroscopia são cureta, bisturi, sonda, pinça com dente e pinça com cesto $^{1,16,23}$. A sonda serve para palpar a superfície articular e manipular tecidos; a pinça com dente para remoção de estruturas; a pinça com cesto para a secção de tecido ${ }^{5,6}$.

Os procedimentos cirúrgicos podem requerer o uso de shavers e/ou unidades eletrocirúrgicas ${ }^{6,16}$. Os primeiros são utilizados para debridar a sinóvia, cartila- 
gem ou osso, os aparelhos de radiofreqüência e eletrocautério são usados para cauterizar vasos e debridar tecidos ${ }^{3}$.

Os instrumentos são inseridos na articulação diretamente por meio de uma incisão ou de uma cânula instrumental 5 .

\section{Técnica Artroscópica Básica}

Para a realização da artroscopia é necessário anestesia geral e técnica asséptica; o paciente é tricotomizado em área semelhante para uma artrotomia e dá-se preferência à utilização de pano de campo impermeável $^{6,16}$ (Figura 1).

O paciente é colocado sobre a mesa de cirurgia e posicionado de acordo com a articulação e abordagem escolhidas ${ }^{5}$. Person ${ }^{10}$ (1986) sugere o uso de aparelho denominado leg holder a fim de facilitar fixaçãoe tração articulares.

Para a distensão articular, é realizada artrocentese com agulha hipodérmica. A saída de líquido sinovial certifica a correta posição da agulha. Na seqüência o líquido é injetado no interior da articulação até que se perceba resistência no êmbolo da seringa ${ }^{23}$.

Para a confecção do portal artroscópico é feita uma pequena incisão na pele e fascia ${ }^{6}$. Em algumas articulações como o joelho, esta incisão pode incluir a cápsula articular ${ }^{5,21}$. Geralmente utiliza-se o trocarte rombo acoplado à cânula para perfurar a cápsula articular. Quando o espaço intra-articular for correta-

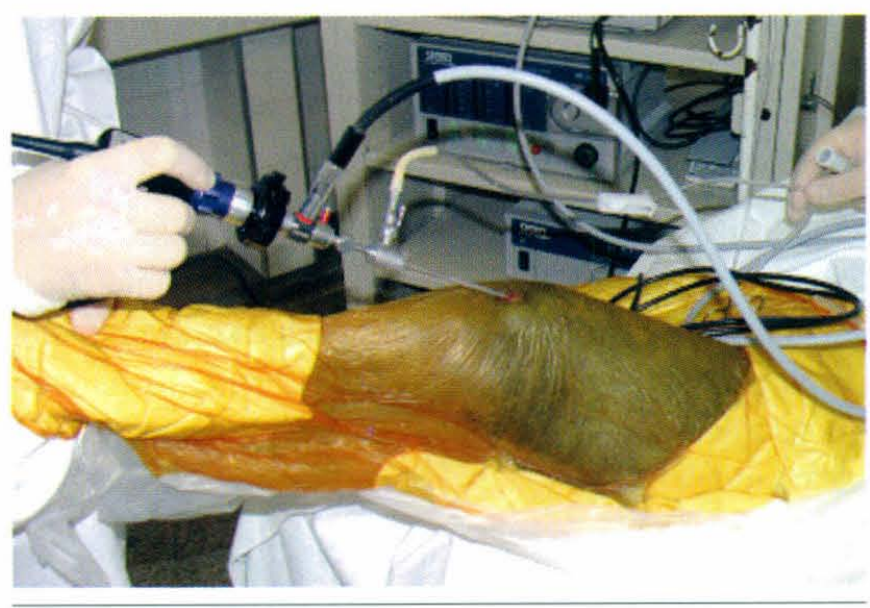

Figura 1 - Artroscopia da articulação do joelho em cão

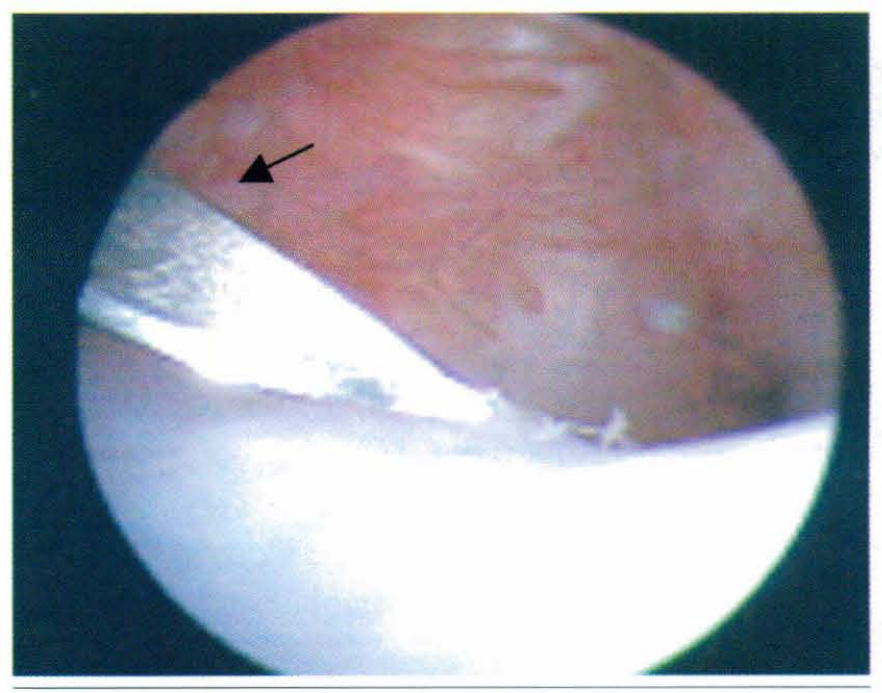

Figura 2 - Visualização por artroscopia de agulha (seta) em articulação de ombro de cão, realização da técnica de triangulação

mente adentrado, o líquido utilizado para distender a articulação fluirá pela cânula no momento em que o trocarte for removido ${ }^{6,21,22}$.

O portal de drenagem é confeccionado usando uma cânula de drenagem, cateter ou agulha hipodérmica $^{6,23}$. As cânulas podem ser dotadas de fenestrações na extremidade a fim de evitar a obstrução de um único orifício por debrís e sinóvia ${ }^{5}$.

A artroscopia deve ser feita de forma sistemática, procurando observar as estruturas metodicamente, mesmo quando o diagnóstico for óbvio ${ }^{8}$.

Para a criação do portal instrumental, pode-se lançar mão do método de triangulação; que corresponde à técnica pela qual se dispõe o artroscópio e o instrumento em ângulos convergentes ${ }^{5,21-23}$. Uma agulha hipodérmica é introduzida no interior da articulação de forma que a sua ponta encontre a ponta do artroscópio (Figura 2) e, sendo a sua posição satisfatória para o objetivo proposto, confecciona-se pequena incisão cutânea no local determinado pela agulha e introduz-se o trocarte rombo (ROCHAT ${ }^{6}, 2001$; McCARTHY $^{16}$, 1999; PERSON $\left.{ }^{24}, 1985\right)$.

O uso de cânulas (Figura 3) para o portal de instrumentos é contraditório entre os autores. Embora a maioria dos artroscopistas prefira introduzir os instrumentos sem utilizá-las, McCarthy ${ }^{16}$ (1999) releva que seu uso é importante em regiões de maior muscu- 


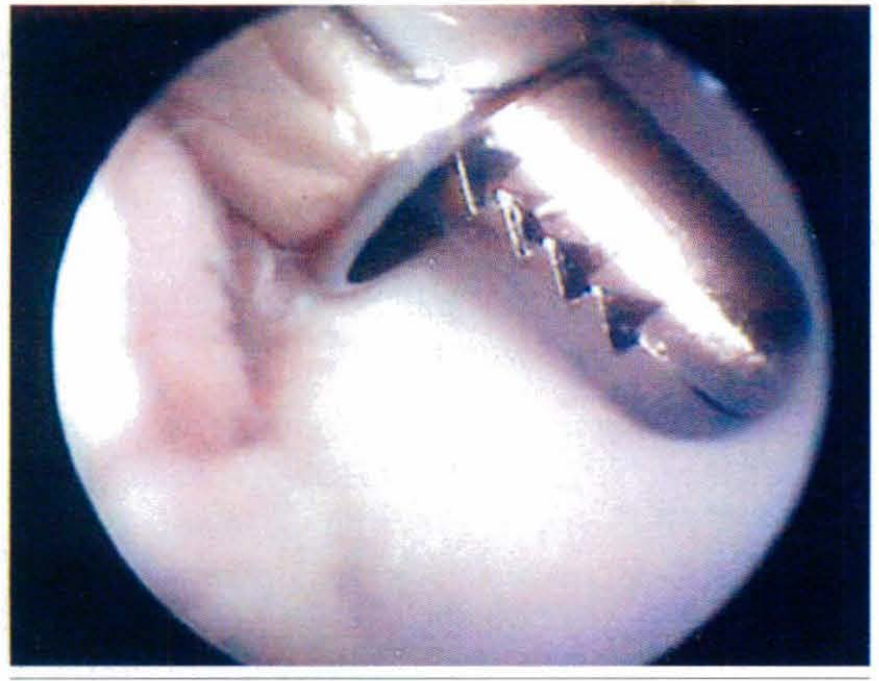

Figura 3 - Articulação do ombro de cão com presença de pinça e cânula em portal instrumental

latura como o portal caudal na articulação do ombro. Os autores van Bree; van Ryssen ${ }^{1}$ (1998) e Rochat ${ }^{6}$ (2001) consideram que as cânulas são grandes para os cães, restringindo o movimento e a manipulação dos instrumentos dentro dos pequenos espaços articulares, e o fato de a sua parede ser rígida torna a remoção dos corpos livres ou fragmentos de cartilagem difícil ou até impossível.

No final do procedimento, os instrumentos são removidos e as incisões de pele suturadas com fio de náilon em ponto simples separado (McCARTHY ${ }^{16}$, 1999; TOMLINSON ${ }^{21,22}$, 2001) ou sutura mecânica.

\section{Complicações}

A maioria das complicações advém da própria técnica e a incidência diminui com a experiência ${ }^{6,16}$.

Falhas técnicas compreendem inabilidade em criar um portal artroscópico ou de instrumentos satisfatórios e em explorar adequadamente uma articulação, dificuldade no procedimento de triangulação, lesão iatrogênica e deslocamento prematuro do artroscópio (MARTINI et al. $.^{27}, 2002$ ), cânula ou agulha de efluxo (BEALE et al. ${ }^{5}, 2003$ ).

Dentre as lesões iatrogênicas intra-articulares, o trauma de cartilagem (Figura 4) é o mais comum, po- dendo ser uma complicação importante dependendo de sua extensão $0^{5,8,9}$.

As lesões neurovasculares são raras, mas passíveis de ocorrer, principalmente nas porções medial e caudal das articulações do cotovelo e ombro, respectivamente (PERSON ${ }^{10}, 1986$; McCARTHY $\left.{ }^{16}, 1999\right)$.

O extravasamento excessivo do líquido de irrigação para os tecidos periarticulares resulta em distorção dos planos teciduais, perda das referências anatômicas para criação de portais, colapso de cápsula articular e, conseqüentemente, impossibilita o prosseguimento do exame $^{6,9}$. Apesar de esta ser uma complicação importante durante $o$ ato operatório, geralmente não raz conseqüências mais graves para o animal no pós-operatório $^{5,16}$.

Durante o procedimento artroscópico pode haver obstrução do campo de visão por elementos celulares ${ }^{9}$, corpos livres, debrís, sangue e dobras sinoviais hipertrofiadas ou vilos ${ }^{16,28}$. A contínua irrigação associada à manipulação da extremidade do artroscópio em varias direções sobrepuja o problema na maioria das vezes ${ }^{8}$.

O diminuto tamanho de muitas articulações torna o procedimento difícil senão impossível de ser realizado $^{9,17}$. Siemering 9 (1978); Kivumbi; Bennett ${ }^{8}$ (1981) e McCarthy ${ }^{16}$ (1999) afirmam que em cães com menos de 6 kg é impossível examinar todas as estruturas intraarticulares.

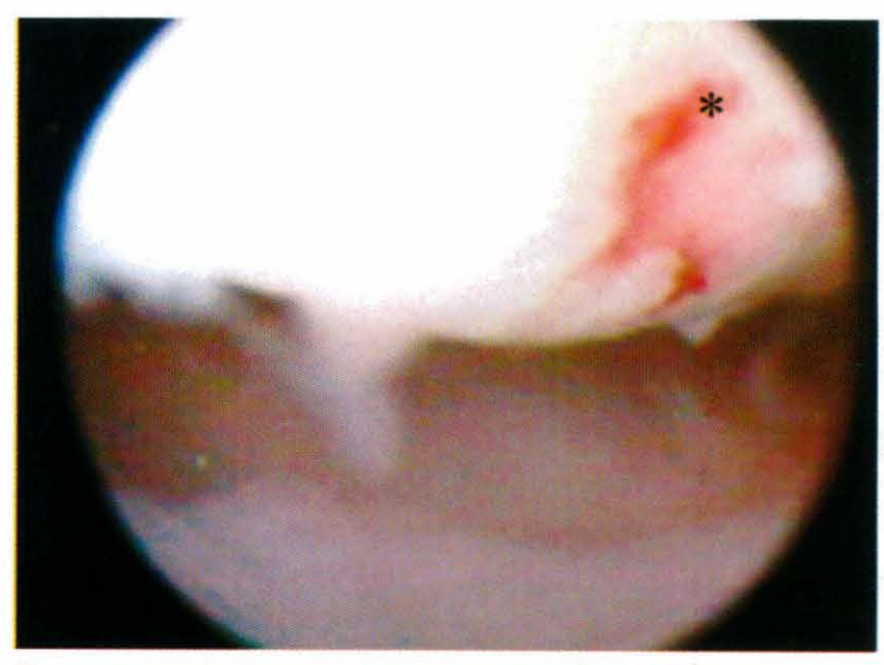

Figura 4 - Lesão iatrogênica de cartilagem (*) com exposição de osso subcondral em côndilo medial de fêmur de cão 
Déficit na fonte de luz, vídeo, câmara ou outro equipamento ou ainda inabilidade de completar o procedimento por via artroscópica podem ser razões para proceder-se à artrotomia ${ }^{16}$.

\section{Articulação do Joelho}

Ojoelhoé uma articulação difícil de ser examinada e não raro leva os iniciantes à frustração e desistência $^{22,23}$. No cão, esta articulação é bem menor quando comparada ao homem e possui o coxim adiposo infrapatelar bastante exuberante (Figura 5), o qual, não raro, precisa ser removido para que se possa examinar a articulação adequadamente ${ }^{15,9,28}$.

Durante a artroscopia é possível o exame da região suprapatelar, patela, bordas trocleares, sulco troclear, côndilos femorais, meniscos, ligamentos meniscais, ligamentos cruzados (Figura 6), côndilos tibiais, tendão do músculo extensor digital longo $0^{8,16,22,24}$ e tendão do músculo poplíteo ${ }^{24}$.

Os portais utilizados para a abordagem desta articulação são infrapatelar lateral e medial para o artroscópio, ou instrumental e suprapatelar lateral ou medial para a cânula de drenagem ${ }^{8,9,16-18,24}$.

Durante o exame da articulação do joelho é imprescindível a abertura adequada de cada compartimen-

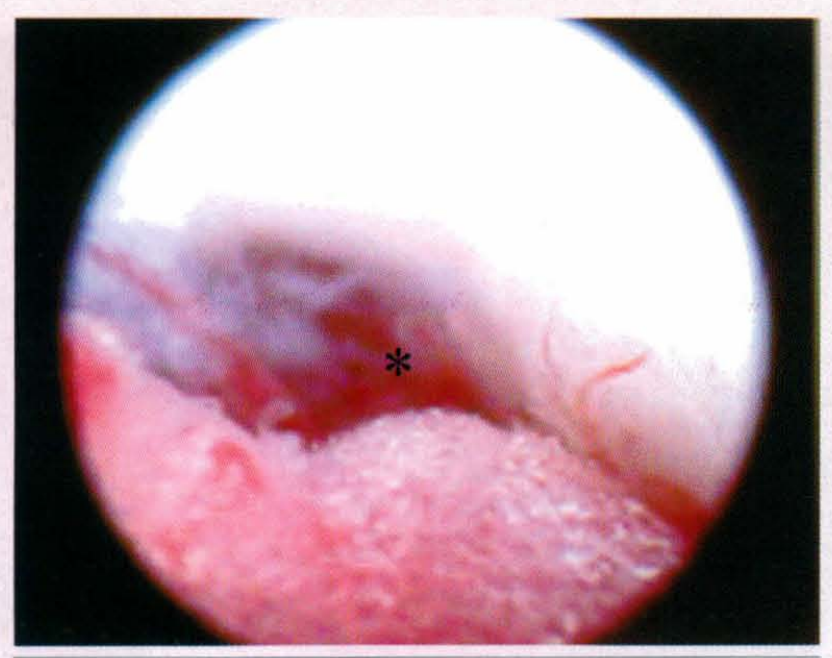

Figura 5 - Articulação do joelho de cão com coxim adiposo infrapatelar $(*)$ to para um exame minucioso das estruturas intra-articulares. Stress varus e valgus, rotação interna e externa e flexão e extensão são utilizados ${ }^{5}$.

A artroscopia da articulação do joelho está indicada em animais com claudicação de membro pélvico e evidência radiográfica de afecção desta como efusão ou doença articular degenerativa ${ }^{16}$.

O tratamento de osteocondrite dissecante (TOMLINSON $^{22}$, 2001; BERTRAND et al. ${ }^{29}, 1997$; McLAUGHLIN et al. ${ }^{30}, 1989$ ), remoção de resquícios de ligamento cruzado cranial rompido, meniscectomias parcial ou total e biópsias são realizados por via artroscópica na espécie canina ( ROCHAT $\left.^{6}, 2001\right)$. A artroscopia permite um diagnóstico mais preciso de todas as estruturas intra-articulares, sendo indicada a sua realização mesmo quando a afecção será corrigida por intermédio de procedimento cirúrgico aberto (BARDET $\left.{ }^{23}, 2000\right)$.

Enquanto no homem a reconstrução do ligamento cruzado cranial via artroscopia é um dos procedimentos mais comuns realizados nesta articulação, no cão, esta afecção representa a maior necessidade e o maior desafio ${ }^{5}$. Foi descrita a sua estabilização artroscópica com material sintético, porém com resultados pouco satisfatórios ${ }^{14}$, e mais recentemente o uso de auto-enxerto ${ }^{3,5}$.

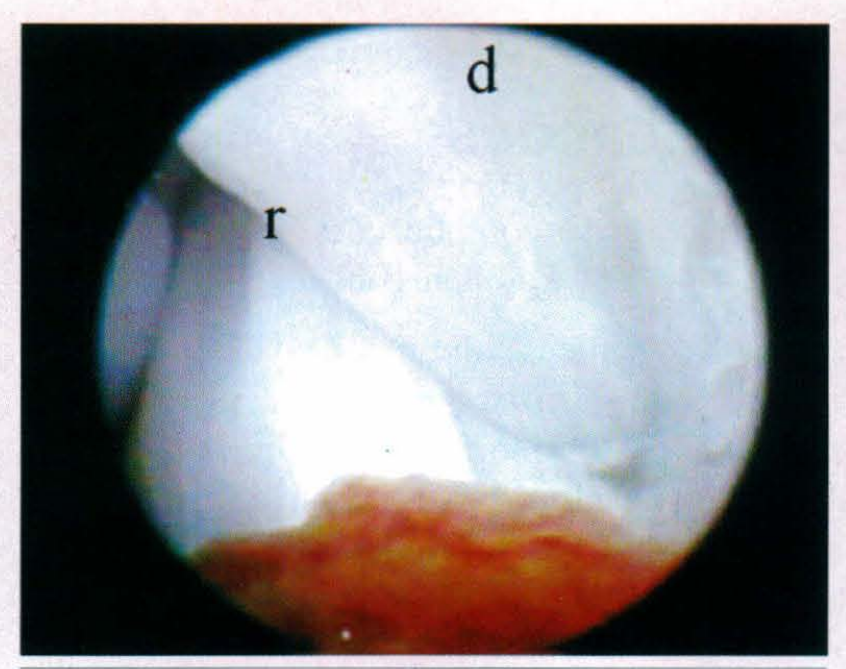

Figura 6 - Ligamentos cruzados cranial (r) e caudal (d) em cão 
Também o debridamento de resquícios de ligamento cruzado cranial, a reparação de fraturas por avulsão do ligamento cruzado cranial ou caudal, osteomielite séptica, osteoartrite, sinovectomia e alguns pacientes portadores de luxação medial de patela podem beneficiar-se do uso da artroscopia ${ }^{5}$.

\section{Articulação do Ombro}

A articulação do ombro, devido a sua anatomia e dimensão, é favorável à artroscopia ${ }^{21,22}$, sendo de eleição para iniciar-se o treinamento da técnica ${ }^{5}$.

Na espécie canina, a artroscopia desta articulação teve grande progresso nestes últimos anos (BARDET ${ }^{31}$, 2002). Sua indicação primária é o diagnóstico e o tratamento da osteocondrite dissecante ${ }^{6,13,22,26}$ e também da instabilidade articular, da tenosinovite ou ruptura do tendão do músculo bíceps braquial $^{33}$, das fraturas articulares ${ }^{34}$, da artrite séptica ${ }^{35}$, das lesões traumáticas e das biópsias ${ }^{5,23}$.

Estruturas passíveis de serem visualizadas são: membrana sinovial, cartilagem articular da cabeça do úmero e cavidade glenóide, ligamento glenoumeral medial, sulco intertubercular, tendão do músculo bíceps braquial, tubérculo supraglenoidal e bolsa articular caudal (PERSON ${ }^{10}$, 1986; van RYSSEN et al. ${ }^{20}$, 1993; GORING; PRINCE ${ }^{25}$, 1987; BARDET $^{31}$, 2002; BARDET $\left.^{32}, 1995\right)$.

Vários portais foram descritos para o artroscópio e instrumental ${ }^{10,13,16,20,25,27,32,36}$. São utilizados 2 ou 3 portais, dependendo do propósito da intervenção. $\mathrm{O}$ portal instrumental cranial geralmente é utilizado quando há necessidade de se intervir nesta porção da articulação (abordagem ao tendão do músculo bíceps braquial), enquanto que para o tratamento de osteocondrite dissecante dá-se preferência para a sua localização mais caudal 5 .

\section{Articulação do Cotovelo}

Segundo Tomlinson ${ }^{22}, 2001$, esta é a articulação mais simples de ser examinada por artroscopia, pois, ao contrário da articulação do ombro, é praticamente isenta de tecidos moles periarticulares. Todavia, devido a sua pequena dimensão interna, o tratamento das afecções torna-se um desafio para o cirurgião ${ }^{5}$.

Os portais artroscópico e instrumental geralmente são estabelecidos na face medial da articulação (McCARTHY ${ }^{16}$, 1999; van RYSSEN et al. ${ }^{20}$, 1993; BARDET $^{37}, 1997$; SAMS $^{38}, 2000$ ), pois fornecem melhor visualização e maior facilidade para o tratamento da área geralmente acometida (van RYSSEN et al. ${ }^{12}$, 1993).

O ligamento colateral medial, tróclea, capítulo, processo coronóide medial, porção central e caudal da cabeça do rádio, processo coronóide lateral, cabeça da ulna, processo ancôneo e membrana sinovial podem ser visualizados durante a artroscopia ${ }^{5,12,38}$.

A indicação mais comum de artroscopia da articulação do cotovelo em cães é o diagnóstico e o tratamento de doenças do desenvolvimento e degenerativas; osteocondrose $e^{1,3,38}$, fragmentação do processo coronóide medial da ulna ${ }^{37}$, não-união do processo ancôneo e osteoartrite 5 .

A artroscopia permite o diagnóstico de fragmentação do processo coronóide medial da ulna e osteocondrite dissecante anterior ao desenvolvimento de osteoartrose, portanto melhorando o prognóstico após o tratamento ${ }^{12,37}$. O diagnostico radiográfico destas afecções geralmente é realizado após o desenvolvimento das alterações degenerativas, segundo Olsson ${ }^{39}$, 1983, e para Voorhout e Hazenwinkel ${ }^{40}, 1987$, exames como tomografia computadorizada ou ressonância magnética nem sempre são disponíveis.

Fraturas articulares podem ser reduzidas com maior acurácia por artroscopia ${ }^{5}$, e Leighton ${ }^{41}, 1971$, também descreve o seu uso para o diagnóstico de ossificação incompleta do côndilo umeral

\section{Outras Articulações}

O estudo da artroscopia em articulações menores como tarso, coxofemoral e carpo são mais recentes ${ }^{5,6}$.

O uso da artroscopia na articulação do tarso consiste principalmente na confirmação diagnóstica e tratamento das lesões de osteocondrose ${ }^{42,43}$, remoção de 
fragmento livre e biópsia ${ }^{6}$. Abordagens descritas por McCarthy ${ }^{16}, 1999$ e Rochat ${ }^{6}, 2001$, são dorsomedial, dorsolateral e plantarolateral, podendo ser usadas isoladas ou associadas.

A artroscopia da articulação coxofemoral foi descrita por Person ${ }^{11}$ em 1989. Ela tem sido feita com o intuito de avaliar a superfície articular anteriormente à realização da osteotomia pélvica tripla em cães jovens displásicos ${ }^{5,16}$.

Biópsia, acesso a fraturas e artrodese são procedimentos que podem ser realizados via artroscopia na articulação do carpo ${ }^{5,6,19}$.

\section{Conclusões}

A artroscopia permite um exame direto, realizado sob magnificação da imagem e iluminação da car- tilagem, membrana sinovial e demais estruturas intraarticulares. Devido ao seu caráter pouco invasivo, ela promove menor trauma tecidual, retorno mais rápido da função do membro e melhor resultado cosmético. Devido às características inerentes da técnica, o seu desenvolvimento no cão trará um acréscimo de informações relevante sobre as afecções articulares que acometem a espécie, tanto no que tange ao diagnóstico como ao tratamento.

\section{Agradecimentos}

Os autores agradecem o apoio da Fundação de Auxílio à Pesquisa do Estado de São Paulo - FAPESP.

\section{Abstract}

Objective: To present advantages and disadvantages of the use of arthroscopy, training, instrumental, technique and complications, as well as specificities and indications for the use of said procedure in shoulder, knee, elbow, ankle, hip and carpus articulations. Data Sources: CAB and MEDLINE, over the last 24 years. Data Synthesis: Though arthroscopy is widely used for diagnosis and treatment of articular diseases in men and horses, it was only in the last years that it has become relevant in dogs. It allows a direct examination of the cartilage, synovial membrane and other intra-articulary structures, that is carried out upon image and lighting magnification. Conclusions: Due to the its little invasive character, arthroscopy promotes lower tissue trauma, a faster return of the function of member and a better cosmetic result.

Keywords: Artroscopy. Joints, surgery. Artroscopes. Dogs.

\section{Resumem}

Objetivo: Presentar las ventajas y desventajas del uso de la artroscopia, entrenamiento, instrumental, técnica y complicaciones, así como de las particularidades e indicaciones del procedimiento en las articulaciones del hombro, rodilla, codo, tarso, caderas y carpo. Fuentes Pesquisadas: CAB y MEDLINE, período retrospectivo de 24 años. Síntesis de los Datos: A pesar de que la artroscopia es ampliamente utilizada para el diagnóstico y el tratamiento de las enfermedades de las articulaciones en el hombre y en el caballo, solamente en los últimos años, viene adquiriendo importancia en los perros. La artroscopia permite un examen directo de cartílago, membrana sinovial y demás estructuras intra-articulares, lo cual se realiza bajo magnificación e iluminación de la imagen. Conclusiones: Debido al carácter de ser poco invasiva la artroscopia causa menor trauma en los tejidos, un retorno más rápido de la función del miembro y un mejor resultado estético.

Palabras-clave: Artroscopia. Articulaciones, cirugía. Artroscopes. Perros. 


\section{Referências}

1. van BREE, H. J. J.; van RYSSEN, B. Diagnostic and surgical arthroscopy in osteochondrosis lesions. Veterinary Clinics of North America: Small Animal Practice, v. 28, p. 161-189, 1998.

2. ROCKWOOD, C. A. Shoulder arthroscopy (editorial). Journal of Bone and Joint Surgery, v. 70-A, p. 639-640, 1988.

3. JOHNSON, A. L.; HULSE, D. A. Diseases of the joints. In: FOSSUM, T. W. Small animal surgery. 2. ed. Saint Louis: Mosby, 2002. p. 1023-1150.

4. McCILWRAITH, W; McCRACKEN, T. Introduction and brief historical: review. In:

Diagnostic and surgical arthroscopy in the horse. 2. ed. Philadelphia: Lea \& Febiger, 1990. p. 1-4.

5. BEALE, B. S. et al. Small animal arthroscopy. Philadelphia: Saunders, 2003. 223p.

6. ROCHAT, M. C. Arthroscopy. Veterinary Clinics of North America: Small Animal Practice, v. 31, p. 761-787, 2001.

7. TAYLOR, R. A. Arthroscopy. In: TAMS, T. R. Small animal endoscopy. 2. ed. Saint Louis: Mosby, 1999. p. 461-470.

8. KIVUMBI, C. W.; BENNETT, D. Arthroscopy of the canine stifle joint. Veterinary Record, v. 109, p. 241-249, 1981.

9. SIEMERING, G. H. Arthroscopy of dogs. Journal of the American Veterinary Medical Association, v. 172, p. 575-577, 1978.

10. PERSON, M. W. Arthroscopy of the canine shoulder joint. Compendium on Continuing Education for the Practicing Veterinarian, v. 8, p. 537-548, 1986.

11. PERSON, M. W. Arthroscopy of the canine coxofemoral joint. Compendium on Continuing Education for the Practicing Veterinarian, v. 11, p. 931-935, 1989.
12. van RYSSEN, B.; van BREE, H. J. J.; SIMOENS, P. Elbow arthroscopy in clinically normal dog. American Journal of Veterinary Research, v. 54, p. 191-198, 1993.

13. van RYSSEN, B.; van BREE, H. J. J.; MISSINNE, S. Successful arthroscopic treatment of shoulder osteochondrosis in the dog. Journal of Small Animal Practice, v. 34, p. 521-528, 1993.

14. PERSON, M. W. Prosthetic replacement of the cranial cruciate ligament under arthroscopic guidance: a pilot project. Veterinary Surgery, v. 16, p. 37-43, 1987.

15. PERSON, M. W. Arthroscopic treatment of osteochondritis dissecans in the canine shoulder. Veterinary Surgery, v. 18, n. 2, p. 175-189, 1989.

16. McCARTHY, T. C. Arthroscopy. In: FREEMAN, L. J. Veterinary endosurgery. Saint Louis: Mosby, 1999. p. 237-250.

17. MILLER, C. W.; PRESNELL, R. K. Examination of the canine stifle: arthroscopy versus arthrotomy. Journal of the American Animal Hospital Association, v. 21, p. 623-629, 1985.

18. van GESTEL, M. A. Arthroscopy of the canine stifle joint. Veterinary Quarterly, v. 7, p. 237-239, 1985.

19. van RYSSEN, B.; van BREE, H. Arthroscopic findings in 100 dogs with elbow lameness. Veterinary Record, v. 140, p. 360-362, 1997.

20. van RYSSEN, B.; van BREE, H. J. J.; VYT, P. Arthroscopy of the shoulder joint in the dog. Journal of the American Animal Hospital Association, v. 29, p.101-105, 1993.

21. TOMLINSON, J. Arthroscopy in dogs: applications. In: WSAVA WORLD CONGRESS, 26., 2001, Vancouver. Proceedings of the Small Animal Veterinary Association Congress ... Vancouver: SOTAL, 2001. p. 641-643. 
22. TOMLINSON, J. Arthroscopy in dogs: basic principles. In: WSAVA WORLD CONGRESS, 26., 2001, Vancouver. Proceedings of the World Small Animal Veterinary Association Congress ... Vancouver: SOTAL, 2001. p. 638-640.

23. BARDET, J. F. L'arthroscopie chez le chien et le chat. Bulletin de I' Académie Vétérinaire de France, v. 73, p. 201-209, 2000.

24. PERSON, M. W. A Procedure for arthroscopic examination of the canine stifle joint. Journal of the American Animal Hospital Association, v. 21, p. 179-186, 1985.

25. GORING, R. L.; PRICE, C. Artroscopical examination of the canine scapulohumeral joint. Journal of the American Animal Hospital Association, v. 23, p. 551-555, 1987.

26. ERIKSON, E.; SEBIK, A. Arthroscopy and arthroscopic surgery in a gas versus a fluid medium. Symposium on Arthroscopic Knee Surgery. Orthopedic Clinics of North America, v. 13, p. 293-298, 1982.

27. MARTINI, F. M.; PINNA, S. A simplified technique for diagnostic and surgical arthroscopy of the shoulder joint in the dog. Journal of Small Animal Practice, v. 43, p. 7-11, 2002.

28. ABERCROMBY, R. Examination of arthroscopy as a clinical tool in small animal practice. Journal of Small Animal Practice, v. 38, p. 174-178, 1997.

29. BERTRAND, S. G. et al. Arthrocopic examination and treatment of osteochondritis dissecans of the femoral condyle of six dogs. Journal of the American Animal Hospital Association, v. 33, p. 451-455, 1997.

30. McLAUGHLIN JR., R. M.; HURTIG, R. M.; FRIES, C. L. Operative arthroscopy in the treatment of bilateral stifle osteochondritis dissecans in a dog. Veterinary and Comparative Orthopaedics and Traumatology, v. 2, p.158-161, 1989.
31. BARDET, J. F. Shoulder diseases in dogs. Veterinary Medicine, v. 97, p. 909-918, 2002.

32. BARDET, J. F. Traitement de l'ostéochondrite disséquante de l'épaule chez le chien par arthroscopie: étude rétrospective de 29 cas. Pratique Médicale et Chirurgie Animale Compareé, v. 30, p. 685-694, 1995.

33. WAL.L; C. R.; TAYLOR; R. Arthroscopic biceps brachii tenotomy as a treatment for canine bicipital. tenosynovitis. Journal of the American Animal Hospital Association, v. 38, p. 169175, 2002.

34. DENEUCHE, A. J.; VIGUIER, E. Reduction and stabilisation of a supraglenoid tuberosity avulsion under arthroscopic guidance in a dog. Journal of Small Animal Practice, v. 43, p. 308-311, 2002.

35. FEARNSIDE, S. M.; PRESTON, C. A. Arthroscopic management of septic polyarthritis in a dog. Australian Veterinary Journal, v. 80, p. 681-683, 2002.

36. BARDET, J. F. Arthroscopie diagnostique de l'épaule chez le chien. Pratique Médicale et Chirurgie Animale Compareé, v. 30, p. 47-54, 1995.

37. BARDET, J. F. Traitement des lesions du processus coronóide par voie arthroscopique mediale: etude retrospective de 24 cas. Pratique Médicale et Chirurgie Animale Compareé, v. 32, p. 57-65, 1997.

38. SAMS, A. E. Canine elbow joint arthroscopy: introduction and description of technique. Compendium on Continuing Education for the Practicing Veterinarian, v. 22, p. 135-145, 2000.

39. OLSSON, S. E. The early diagnosis of fragmented coronoid process and osteochondritis dissecans of the canine elbow joint. Journal of the American Animal Hospital Association, v. 19, p. 616-626, 1983. 
40. VOORHOUT, G.; HAZEWINKEL, H. A. W. Radiographic evaluation of the canine elbow joint with special. reference to the medial humeral condyle and the medial coronoid process. Veterinary Radiology, v. 28, p. 158-165, 1987.

41. LEIGHTON, R. L. Osteochondritis dissecans of the shoulder joint of the dog. Veterinary Clinics of North America: Small Animal Practice, v. 19, p. 391-395, 1971.
42. van RYSSEN, B.; van BREE, H. J. J. Arthroscopic evaluation of osteochondrosis lesions in the canine hock joint: a review of two cases. Journal of the American Animal Hospital Association, v. 28, p. 295-299, 1992.

43. van RYSSEN, B.; van BREE, H. J. J.; VYT, P. Arthroscopy of the canine hock joint. Journal of the American Animal Hospital Association, v. 29, p. 107-115, 1993. 